selbst entwickelt wird. Wie sehr die Auseinandersetzung zwischen den beiden (intern alles andere als kohärenten) Lagern der Federalists und Anti-Federalists dazu beigetragen hat daran erinnert der Eingangsbeitrag von Jörn Ketelhut. Die Federalist Papers sind zweifellos ein schönes Beispiel für die Einsicht, dass es nichts Praktischeres gibt als eine gute Theorie.

Oliver W. Lembcke

\title{
Governance - Erkenntnisse und Ideen zu einem anerkannt unerkannten Großkonzept
}

Benz, Arthur und Nicolai Dose (Hrsg.): Governance - Regieren in komplexen Regelsystemen. Eine Einführung, 2. aktualisierte und veränderte Auflage, VS Verlag für Sozialwissenschaften, Wiesbaden 2010, 280 Seiten, $€ 26,95$.

Norris, Pippa: Making Democratic Governance Work. How Regimes Shape Prosperity, Welfare, and Peace, Cambridge University Press, Cambridge 2012, 296 Seiten, $\$ 29.99$ (Paperback).

Schuppert, Gunnar Folke: Der Rechtsstaat unter den Bedingungen informaler Staatlichkeit. Beobachtungen und Überlegungen zum Verhältnis formeller und informeller Institutionen, Nomos Verlagsgesellschaft, Baden-Baden 2011a, 193 Seiten, € 34,-.

Schuppert, Gunnar Folke: Alles Governance oder was?, Nomos Verlagsgesellschaft, Baden-Baden 2011b, 46 Seiten, $€ 19$,-

Der Governance-Begriff hat in der wissenschaftlichen Diskussion der vergangenen 20 Jahre eine rasante Karriere hinter sich. Inzwischen ist man leicht geneigt, die von Werner Jann in Bezug auf die Policy- und Verwaltungsforschung formulierte Frage „Alles Governance, oder was?" 1 auch ganz allgemein zu stellen. Pippa Norris (S. 15) formuliert: „Governance ... has become intellectually fashionable in recent years, yet it remains a complex and contested concept that is open to multiple meanings, often with a long and ever-growing shopping list of potential attributes. "Vier aktuelle Bücher nähern sich dem Thema auf unterschiedlichen Wegen. Einen vielfältigen Überblick und gründlichen Einstieg bietet der in zweiter, überarbeiteter Auflage erschienene Sammelband von Arthur Benz und Nicolai Dose. Die beiden Monographien decken hingegen zwei zentrale Schwerpunkte der Governance-Forschung ab: einerseits die eher der Entwicklungszusammenarbeit entstammende angewandte angelsächsische Perspektive bei Pippa Norris und andererseits die staatstheoretische deutsche Blickweise bei Gunnar Folke Schuppert (2011a). Letzterer hat zudem einen schmalen Essayband verfasst, der die Forschung zum Thema kenntnisreich sortiert und in flottem Stil lesenswert bilanziert (Schuppert 2011b).

Der Sammelband von Arthur Benz und Nicolai Dose wird vom Verlag als Lehrbuch geführt und beginnt mit einer gründlichen Begriffsdiskussion durch die beiden Herausgeber. Dabei wird die ursprüngliche Begriffsentwicklung aus der frühen Institutionenökonomie

1 Werner Jann, Praktische Fragen und theoretische Antworten: 50 Jahre Policy-Analyse und Verwaltungsforschung, in: PVS, 50. Jg. (2009), H. 3, S. 476 - 505, S. 494. 
von Ronald Coase (1937) in die Neokorporatismus-Diskussion der 1980er Jahre hin zur politischen Praxis der Entwicklungspolitik (Good Governance) nachgezeichnet. Das explosionsartige Wachstum des Begriffes begann erst im Anschluss: Governance wurde zunächst in der Policyforschung und dann in Regierungslehre und Verwaltungswissenschaft sowie zur Bezeichnung internationaler, supranationaler und transnationaler Prozesse (Global Governance) verwendet. Als „konstanter Begriffskern“ von Governance sehen die Verfasser dabei, das „Steuern und Koordinieren“, das auf institutionalisierten Regeln sowie faktischen Interaktionsmustern beruhe und Organisationsgrenzen überschreite, insbesondere auch die klassische Unterscheidung von Staat und Gesellschaft überwinde (S. 25 f.). Renate Mayntz geht in ihrem Beitrag noch weiter und definiert Governance als „Gesamtheit aller in einem Gemeinwesen bestehenden und miteinander verschränkten Formen der kollektiven Regelung gesellschaftlicher Sachverhalte“ (S. 38). Sie zeichnet die Entwicklung von der Steuerungstheorie zur Governance-Forschung nach und warnt zugleich vor deren „Problemlösungsbias“; denn was eigentlich banal ist, gerät leicht in Vergessenheit: „Tatsächlich geht es in der Politik keineswegs immer und primär um Aufgabenerfüllung, Leistungserbringung und Problemlösung, sondern oft in erster Linie um Gewinn und Erhalt politischer Macht" (S. 46).

In den weiteren Beiträgen werden verschieden attributierte Ausprägungen von Governance jeweils einführend dargestellt. So gibt es Kapitel zu Regional Governance, Governance in der Europäischen Union, Global Governance und so weiter. In einem gründlichen Überblick zur Multilevel Governance zeigt Arthur Benz auf, dass kaum Wissen darüber vorhanden ist, „unter welchen Bedingungen Governance in Mehrebenensystemen effektiv funktioniert“; insbesondere die Rolle von Parlamenten, Parteien und Verbänden in diesen Strukturen müsse besser erforscht werden, um auch die Frage nach der demokratischen Legitimation beantworten zu können (S. 131). Das Kapitel zur Corporate Governance von Dagmer Eberle ist ebenfalls lesenswert und entstammt einer ganz eigenen organisationswissenschaftlichen Forschungstradition. Nicht aus der Wissenschaft, sondern aus der Praxis stammt die Diskussion über Good Governance, die Roland Czada in seinem Beitrag darstellt. Dabei weist er skeptisch darauf hin, dass viele Programme auf die Errichtung politisch-administrativer Strukturen zielen, die aber zugleich wichtige Voraussetzung für deren Gelingen seien: „Dies gleicht dem Versuch, ein Schiff in stürmischer See zu bauen“ (S. 219).

Struktur, Qualität, disziplinärer Zugang und Schreibstil der Sammelband-Beiträge variieren, und es gibt einige Wiederholungen; beinahe jeder Autor beginnt mit einer allgemeinen Begriffsdefinition. Ein klassisches Lehrbuch ist dieser Sammelband also nicht, einen ordentlichen Überblick bietet er aber ohne Frage. Die zum Einstieg herausgestellte Vielfalt des Governance-Begriffes wird somit in der Gesamtschau eindrücklich demonstriert - und in ihrem Fazit kommen die Herausgeber zu dem Ergebnis, dass Governance wohl auch in Zukunft schwammig bleiben werde und die Entwicklung einer einheitlichen GovernanceTheorie kaum in Aussicht stehe (S. 274).

Wozu ist dann der Governance-Begriff überhaupt gut? Gunnar Folke Schuppert stellt in seinem Essay-Band („Alles Governance oder was?“) sieben Funktionen des GovernanceKonzeptes zur Diskussion. In seiner (1) Brückenfunktion erlaube und erleichtere es den intra- und interdisziplinären Austausch, als (2) Reformstrategie sei es zum Verständnis politischer Praxis hilfreich, als (3) Schlüsselbegriff schaffe es einen „ganzheitlichen Blick auf die gesamte Bandbreite der Steuerungsmodi“, wobei Akteure wie Institutionen einfließen (S. 20). Eine wichtige weitere Funktion sei, dass (4) Governance auf die Meta-Ebene ziele, dabei auf eine sektor- wie politikfeldübergreifende Perspektive verweise und somit auch zur 
Analyse von Mehrebenenverflechtung gut geeignet sei. So helfe der Begriff auch (5) die Staatszentriertheit beim Verständnis politischer Prozesse zu überwinden. Weiterhin sei er bei der (6) Betrachtung länderübergreifender Verrechtlichungsprozesse hin auf dem Weg zu „transnational governance“ nützlich. Schließlich sei (7) zu fragen, ob der Governance-Begriff nicht allzu oft dazu verwendet werde, eurozentristische - oder allgemeiner „westliche“ Institutionen in die restliche Welt zu übertragen. Schuppert betont, dass Governance sinnvollerweise als rein analytisches Konzept zu verstehen sei; hingegen sei die „Prominenz des ,Good Governance'-Konzepts für die Governance-Forschung wahrlich ein Danaer-Geschenk" (S. 43).

Der zweite Band von Schuppert (2011a) zum „Rechtsstaat und den Bedingungen informaler Staatlichkeit“ ist keine eigenständige Studie, sondern eher als anregendes Lesebuch zu verstehen, das bisweilen Werkstattcharakter hat und in einigen Details etwas unfertig wirkt. Doch ohne Frage bietet es einen kenntnisreich geleiteten Rundflug über das unübersichtliche Terrain von informaler Staatlichkeit, des Verhältnisses formeller und informeller Institutionen zueinander und sich daraus ergebende Konsequenzen für das Verständnis von Rechtsstaatlichkeit. Schuppert scheut sich nicht davor, andere Autoren sehr ausführlich in direkten Zitaten zu Wort kommen zu lassen; auch an deren flotter Bewertung („enttäuschend“, „korsetthaft") spart er nicht. Im Kern kommt er zu dem Ergebnis, dass formelle und informelle Institutionen sich ergänzen können und zwischen ihnen kein kategorialer, sondern nur ein gradueller Unterschied bestehe: Für das angemessene Verständnis politischer Systeme seien stets beide in ihrer Verknüpft- und Verwobenheit zu berücksichtigen. Normativ problematisch werde es, wenn formelle und informelle Regeln gegenläufig seien, denn dies könne den Rechtsstaat beschädigen. Viele Beispiele begleiten die Darstellung: Vom Deutschen Corporate Governance Kodex zu arabischen Familienclans in Berlin-Neukölln über Favelas in Rio bis zu lokalen Akteuren in Marokko und Jordanien geht die Reise, um zu zeigen, wie formelle und informelle Institutionen sich gegenseitig ergänzen, aber auch überformen können. An disziplinären Grenzen macht der Jurist Schuppert nicht halt, er nimmt vieles aus Soziologie, Ethnologie, Volkswirtschaftslehre und Politikwissenschaft auf. Viele Abbildungen verdeutlichen die Zusammenhänge, und der großzügig verwendete Fettdruck erleichtert die Orientierung. Kritisieren kann man allenfalls, dass die Argumentation in Teilen recht anekdotisch bleibt und wenig systematisch gelingt. Doch ohne Frage handelt es sich um ein interessantes Lesebuch eines belesenen Autors.

Strukturiert und systematisch prüfend geht hingegen Pippa Norris in ihrer Studie zu „Democratic Governance“ vor. Ihr Ausgangspunkt ist die in der Entwicklungszusammenarbeit sehr praxisrelevante Frage, ob man besser mit der Etablierung demokratischer Prozesse oder mit dem Aufbau funktionierender Verwaltung beginnen solle. Um eine Antwort hierauf zu finden, kommen sowohl quantitative als auch qualitative Analysen zum Einsatz, die auf ihrer vielleicht etwas großspurig benannten vereinheitlichten Theorie („unified theory“) aufbauen, die beide Komponenten von Entwicklung - Verwaltung und Demokratie - berücksichtigt. Norris stellt zunächst ein einfaches Raster vor, mit dem die Dimensionen von Demokratisierung und Verwaltungsaufbau einbezogen werden können (S. 39). Basierend auf vorliegenden Indizes zur Demokratie- und Verwaltungsqualität werden entsprechende Zuordnungen von über 100 Ländern vorgenommen, was eine kurvilineare Beziehung ergibt. Anschließend setzt Norris diese ins Verhältnis zu den Wirkungen auf Wohlstand, soziale Sicherung und Frieden, wobei jeweils mehrere Indikatoren berücksichtigt und andere naheliegende intervenierende Variablen (zum Beispiel BIP) herausgerechnet werden. Ergänzend 
werden ausführliche Fallstudien zu einzelnen Ländern vorgestellt. Es zeigt sich, dass demokratische Systeme mit einer funktionierenden Verwaltung stets am besten dastehen. Norris schlussfolgert, was sie auch schon zum Einstieg ihrer Arbeit gewissermaßen als Prämisse formuliert hat: Es ist nicht ausreichend, nur einen Schwerpunkt in Reformen zu verfolgen. „A fine balance needs to be struck so that democracy and state capacity are developed simultaneously“ (S. 192).

In der Zusammenschau unterschiedlicher aktueller Texte zeigt sich dreierlei: Der Governance-Begriff bleibt mehrdeutig und wird sehr vielfältig verwendet. Folglich muss zum Verständnis politisch-administrativer Prozesse der Blick über die klassischen organschaftlichen Akteure hinausgehen - was aber keine bahnbrechende Erkenntnis ist. Und schließlich betont er die Bedeutung des Informalen neben dem Formalen; auch dies ist für Politikwissenschaftler nicht neu. Eine Gefahr besteht in der normativen Überhöhung des Begriffes und in der analytischen Umkehrung der Diagnose: Auch wenn Staatlichkeit sich wandelt, ist sie zu einem Großteil weiterhin in klassischer Form wirksam. Ohne Frage hilft der Governance-Begriff als analytische Brille, die Realität wahrzunehmen - doch darf man nicht vergessen, auch die „alten“ Brillen wie Gewaltenteilung, Demokratie, Macht aufzusetzen. Aus dieser Kombination erhält man am ehesten ein geschärftes Bild der sich ständig verändernden Realität.

Sven T. Siefken

\section{Regieren in Partnerschaft - eindrucksvoller Blick hinter die Kulissen in Paris und Bonn}

Waechter, Matthias: Helmut Schmidt und Valéry Giscard d'Estaing - Auf der Suche nach Stabilität in der Krise der 70er Jahre, Studien der Helmut und Loki Schmidt-Stiftung, Band 6, Edition Temmen, Bremen 2011, 168 Seiten, € 14,90.

Gut ein Jahr vor dem 50. Jahrestag der Unterzeichnung des deutsch-französischen Kooperationsvertrages am 22. Januar 1963 („Élysée-Vertrag“) legte Matthias Waechter, Direktor des Nizzaer Institut des Hautes Études Internationales und Privatdozent am Historischen Seminar der Universität Freiburg, eine 168-seitige Studie über das Tandem Helmut Schmidt und Valéry Giscard d'Estaing vor. Beide gehören neben Charles de Gaulle und Konrad Adenauer sowie François Mitterrand und Helmut Kohl zu den Architekten der binationalen Zusammenarbeit. Beide Staatsmänner übernahmen nahezu gleichzeitig die wichtigsten politischen Ämter in ihren Ländern: Schmidt nach Willy Brandts Rücktritt im Mai 1974 und Giscard d'Estaing nach Georges Pompidous Tod im Juni desselben Jahres. Schon vorher hatten sie sich als Finanzminister kennen- und schätzen gelernt. Das Ende ihrer Amtszeiten fiel auch nicht weit auseinander: Mai 1981 für den Staatspräsidenten und Oktober 1982 für den Bundeskanzler.

Gegliedert ist Waechters verdienstvolles Werk in fünf Kapitel. Nach der Einleitung beleuchtet er den persönlichen und politischen Werdegang beider: Der Deutsche stammte aus kleinbürgerlichem Milieu, sein französischer Partner aus dem Großbürgertum mit Schloss in der Auvergne. Der erste Hauptteil der Studie firmiert unter dem Titel „Kooperation in der internationalen Krise“ nach dem ersten Ölpreisschock im Herbst 1973. Beide Staatsmänner wurden „von unterschiedlichen öffentlichen Erwartungen und Wahrnehmungen 